Int. Int. Journal of Math. Analysis, Vol. 7, 2013, no. 5, 213 - 217

\title{
Face Detection and Visual Landmarks Approach to Monitoring of the Environment
}

\author{
Anna Gorbenko \\ Department of Intelligent Systems and Robotics \\ Ural Federal University \\ 620083 Ekaterinburg, Russia \\ gorbenko.ann@gmail.com \\ Vladimir Popov \\ Department of Intelligent Systems and Robotics \\ Ural Federal University \\ 620083 Ekaterinburg, Russia \\ Vladimir.Popov@usu.ru
}

\begin{abstract}
Many problems of technical vision received a lot of attention recently. In this paper, we consider the problem of monitoring of passenger flows.

To solve this problem we use Haar cascades.
\end{abstract}

Mathematics Subject Classification: 42C40, 65T60

Keywords: Haar cascades, technical vision, visual landmarks

Many problems of technical vision received a lot of attention recently (see e.g. [1] - [3]). In particular, we can mention problems of robot visual navigation (see e.g. [4] - [11]). In this paper, we consider the problem of monitoring of passenger flows. Note that one of the conditions for effective functioning of the transport complex is the systematic monitoring of passenger flows. We can use passenger detection to solve this task. Haar cascades are traditionally used in the real-time face detection (see e.g. [12]).

Note that visual recognition problems can be divided into two main categories, object recognition and object class recognition. It is clear that the former involves the efficient detection of a specific object with certain visual attributes. In the latter category we try to learn an internal model that corresponds to all objects of a class and we are not looking for a specific instance 


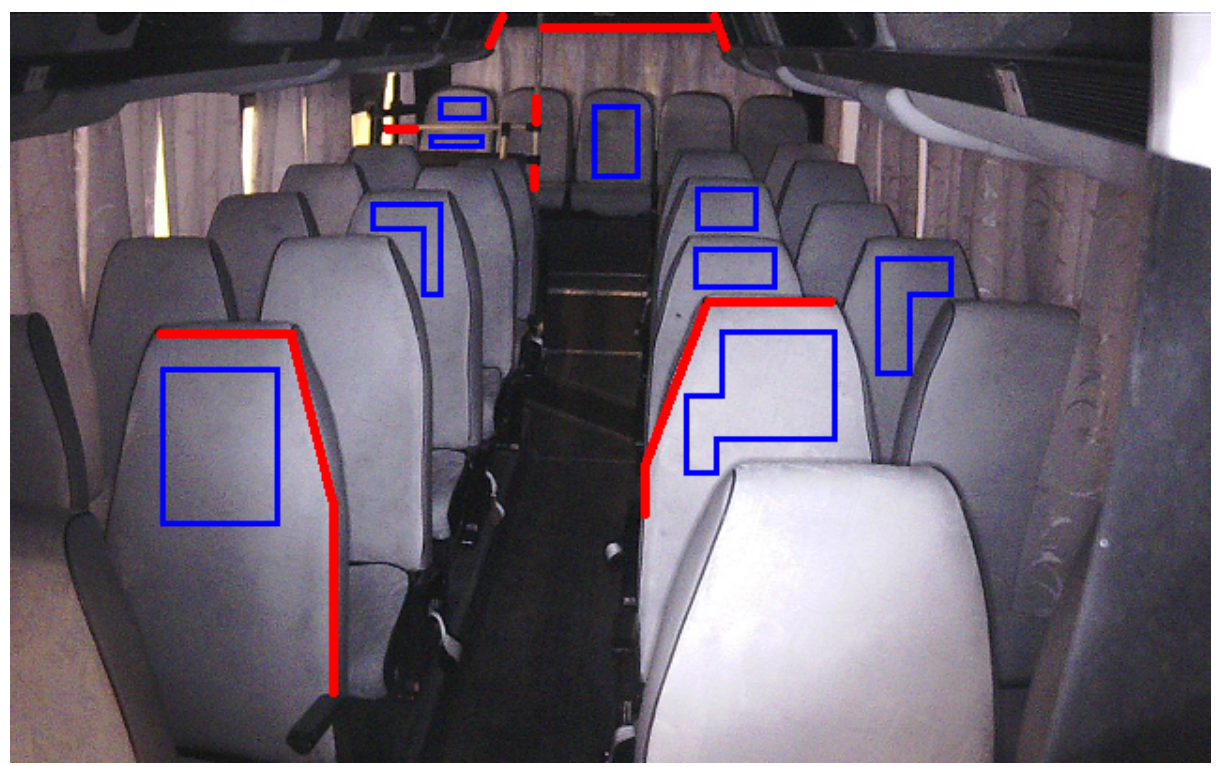

Figure 1: Examples of basic (blue) and additional (red) landmarks.

within a class. Clearly, since detection of any specific passenger is very hard problem, usage of passenger class recognition is more preferable. However, in this case, we need a permanent monitoring of the environment. Note that different problems of landmarks placement are extensively studied recently (see e.g. [13] - [19]). In particular, to solve our problem we can use a visual landmarks approach. As visual landmarks we consider chairs, rail, and some other points of the bus salon (see Figure 1).

We can use simple visual landmarks model. Also, we can consider an intelligent visual landmarks model which allows us to determine quality of visual landmarks and use proper refreshment of visual landmark system.

In our experiments, we consider videos that have been received from one bus camera. As experimental data, we have selected a representative set of samples. We consider the following characteristics of the set, the presence of different numbers of passengers, levels of illumination, levels of camera focus, levels of camera calibration. This characteristics we consider in the following order, increasing the number of passengers and reducing the level of illumination, camera focus, and camera calibration. We obtain a sequence of files

$$
F[0], F[1], \ldots, F[11] \text {. }
$$

Using visual observation we have obtained the exact number $N u m(F[i])$ of passengers for each $F[i]$. For each video $F[i], 1 \leq i \leq 11$, we consider only images $F[i, j], 0 \leq j \leq 9$. For any image $X$ and detector $Y$, let $R(X, Y)$ be the result of detection of passengers on the image $X$ by the detector $Y$.

We consider Haar cascades $H[2], H[3], H[4], H[5]$ from OpenCV 2.1 
haarcascade_frontalface_alt.xml, haarcascade_profileface.xml, haarcascade_frontalface_alt_tree.xml, haarcascade_frontalface_alt2.xml,

respectively), Haar cascade based algorithm $H[0]$ which uses simple visual landmarks model, and Haar cascade based algorithm $H[1]$ which uses intelligent visual landmarks model. Let

$$
N=\frac{\sum_{i=0}^{11} N u m(F[i])}{12} .
$$

Let

$$
P[n]=\frac{\sum_{i=0}^{11} \sum_{j=0}^{9} R(F[i, j], H[n])}{120} .
$$

Selected experimental results are given in Figure 2.

\begin{tabular}{|l|llllll|}
\hline$n$ & 0 & 1 & 2 & 3 & 4 & 5 \\
\hline$P[n]$ & 3.91 & 4.2 & 2.98 & 2.01 & 2.33 & 3.81 \\
\hline
\end{tabular}

Figure 2: Experimental results for $N=10.33$.

\section{References}

[1] A. Gorbenko and V. Popov, On Face Detection from Compressed Video Streams, Applied Mathematical Sciences, 6 (2012), 4763-4766.

[2] A. Gorbenko and V. Popov, Usage of the Laplace Transform as a Basic Algorithm of Railroad Tracks Recognition, International Journal of Mathematical Analysis, 6 (2012), 2413-2417.

[3] A. Gorbenko, A. Lutov, M. Mornev, and V. Popov, Algebras of Stepping Motor Programs, Applied Mathematical Sciences, 5 (2011), 16791692.

[4] A. Gorbenko and V. Popov, Self-Learning Algorithm for Visual Recognition and Object Categorization for Autonomous Mobile Robots, Lecture Notes in Electrical Engineering, 107 (2012), 1289-1295. 
[5] A. Gorbenko and V. Popov, Anticipation in Simple Robot Navigation and Learning of Effects of Robot's Actions and Changes of the Environment, International Journal of Mathematical Analysis, 6 (2012), 2747-2751.

[6] A. Gorbenko and V. Popov, The c-Fragment Longest Arc-Preserving Common Subsequence Problem, IAENG International Journal of Computer Science, 39 (2012), 231-238.

[7] A. Gorbenko and V. Popov, Robot Self-Awareness: Occam's Razor for Fluents, International Journal of Mathematical Analysis, 6 (2012), 14531455 .

[8] A. Gorbenko and V. Popov, The Force Law Design of Artificial Physics Optimization for Robot Anticipation of Motion, Advanced Studies in Theoretical Physics, 6 (2012), 625-628.

[9] A. Gorbenko, V. Popov, and A. Sheka, Robot Self-Awareness: Exploration of Internal States, Applied Mathematical Sciences, 6 (2012), 675688.

[10] A. Gorbenko, V. Popov, and A. Sheka, Robot Self-Awareness: Temporal Relation Based Data Mining, Engineering Letters, 19 (2011), 169-178.

[11] A. Gorbenko, V. Popov, and A. Sheka, Localization on Discrete Grid Graphs, Lecture Notes in Electrical Engineering, 107 (2012), 971-978.

[12] P. Viola and M. Jones, Rapid object detection using a boosted cascade of simple features, Proceedings of the 2001 IEEE Computer Society Conference on Computer Vision and Pattern Recognition, (2001), I-511-I-518.

[13] A. Gorbenko and V. Popov, Computational Experiments for the Problem of Selection of a Minimal Set of Visual Landmarks, Applied Mathematical Sciences, 6 (2012), 5775-5780.

[14] A. Gorbenko and V. Popov, The Problem of Selection of a Minimal Set of Visual Landmarks, Applied Mathematical Sciences, 6 (2012), 4729-4732.

[15] A. Gorbenko and V. Popov, A Real-World Experiments Setup for Investigations of the Problem of Visual Landmarks Selection for Mobile Robots, Applied Mathematical Sciences, 6 (2012), 4767-4771.

[16] A. Gorbenko and V. Popov, On the Problem of Placement of Visual Landmarks, Applied Mathematical Sciences, 6 (2012), 689-696.

[17] A. Gorbenko, M. Mornev, V. Popov, and A. Sheka, The Problem of Sensor Placement, Advanced Studies in Theoretical Physics, 6 (2012), 965-967. 
[18] A. Gorbenko and V. Popov, On the Problem of Sensor Placement, Advanced Studies in Theoretical Physics, 6 (2012), 1117-1120.

[19] A. Gorbenko, M. Mornev, V. Popov, and A. Sheka, The problem of sensor placement for triangulation-based localisation, International Journal of Automation and Control, 5 (2011), 245-253.

Received: September, 2012 